\title{
Reversion of trichostatin A resistance via inhibition of the Wnt signaling pathway in human pancreatic cancer cells
}

\author{
BENQUAN WANG $^{1}$, QIAN ZOU $^{1}$, MENG SUN $^{1}$, JINGFENG CHEN $^{1}$, TIANYANG WANG ${ }^{1}$, \\ YONGHENG BAI $^{1,2}$, ZONGJING CHEN $^{1}$, BICHENG CHEN ${ }^{1,2}$ and MENGTAO ZHOU ${ }^{1}$ \\ ${ }^{1}$ Department of Surgery The First Affiliated Hospital, Wenzhou Medical University, Wenzhou, Zhejiang 325000; \\ ${ }^{2}$ Zhejiang Provincial Top Key Discipline in Surgery, Wenzhou Key Laboratory of Surgery, Department of Surgery, \\ The First Affiliated Hospital, Wenzhou Medical University, Wenzhou, Zhejiang 325000, P.R. China
}

Received June 16, 2014; Accepted August 22, 2014

DOI: $10.3892 /$ or.2014.3476

\begin{abstract}
Drug resistance is a major impediment to successful chemotherapy in pancreatic cancer (PC) patients. We investigated the effect of $\mathrm{Wnt} / \beta$-catenin signaling inhibition by wnt-c59 on chemoresistance in a trichostatin A-resistant Panc-1 cell line (Panc-1/TSA). Panc-1/TSA cells were treated with the Wnt/ $\beta$-catenin signaling inhibitor wnt-c59 $\left(10 \mu \mathrm{mol} \cdot \mathrm{l}^{-1}\right)$ and $/$ or trichostatin A (TSA; $10 \mu \mathrm{mol} \cdot \mathrm{l}^{-1}$ ) for $24 \mathrm{~h}$. CCK-8 assay was utilized to analyze the interactive effect of TSA and wnt-c59 on induction of apoptosis of the Panc-1/TSA cells Cell apoptosis was measured by flow cytometry. Real-time PCR and western blotting were used to assess Wnt/ $\beta$-catenin signaling, epithelial-mesenchymal transition (EMT) and multidrug resistance (MDR). Real-time cell analysis (RTCA) was used to detect the cell migration ability. After wnt-c59 treatment for $24 \mathrm{~h}$, relative genes and transcriptional targets of Wnt $/ \beta$-catenin signaling were downregulated $(\mathrm{P}<0.05)$. CCK-8 assay indicated that the combination of TSA and wnt-c59 had a synergistic effect on induction of Panc-1/TSA cell apoptosis. As detected by FACS, cell apoptosis rates increased significantly $(\mathrm{P}<0.05)$. The results of RTCA showed that the cell indices of the control group, wnt-c59 group, TSA group and TSA+wnt-c59 combination group were 1.2842 \pm 0.0257 , $1.2155 \pm 0.0282,1.2533 \pm 0.0194$ and $0.8541 \pm 0.0250$, respectively. In accordance, MMP-9 protein in the wnt-c59 treatment groups was decreased compared to the non-wnt-c59 treatment
\end{abstract}

Correspondence to: Professor Mengtao Zhou or Dr Bicheng Chen, Department of Surgery, The First Affiliated Hospital, Wenzhou Medical University, 2 Fuxue Lane, Wenzhou, Zhejiang 325000, P.R. China

E-mail: bisonch@163.com

E-mail: chenbicheng@hotmail.com

Abbreviations: APC, adenomatous polyposis coli; PC, pancreatic cancer; MDR, multidrug resistance; P-gp, P-glycoprotein; GSK3 $\beta$, glycogen synthase kinase 3 $\beta$; TCF/LEF1, T cell factor/lymphoid enhancer-binding factor 1

Key words: drug resistance, epithelial-mesenchymal transition, pancreatic cancer, trichostatin $\mathrm{A}, \mathrm{Wnt} / \beta$-catenin signaling pathway groups. Meanwhile, E-cadherin protein was upregulated and vimentin protein was downregulated, both of which are characteristic markers of EMT. Chemoresistant gene MDR1 and P-glycoprotein (P-gp) in the wnt-c59 treatment groups had a reduced expression compared to the non-wnt-c59 treatment groups. This study revealed that TSA sensitivity, migration ability, and the EMT phenotype in Panc-1/TSA cells were reversed following Wnt/ $\beta$-catenin signaling inhibition.

\section{Introduction}

Over the past three decades, pancreatic cancer (PC) has remained one of the most lethal cancers worldwide, leading to an estimated 227,000 deaths annually (1). Despite some efforts to improve diagnosis and treatment strategies (including surgery, chemotherapy, radiation therapy and immunotherapy), the overall 5-year survival rate of all PC patients is dismal, at approximately $5 \%$. At present, less than $20 \%$ of PC patients are indicated for surgery. Chemotherapy has become the standard treatment of locally advanced and metastatic PC patients when disregarding surgical resection as the optimal therapeutic option $(2,3)$. However, patients undergoing continued chemotherapy ultimately develop chemoresistance (4). Hence, effective therapeutic strategies for increasing the sensitivity of chemotherapy or reducing the multidrug resistance (MDR) of PC cells are urgently needed to improve patient survival.

To date, the principal mechanisms of chemoresistance may include increased efflux, activation of detoxifying systems, DNA repair, and failure to undergo apoptosis (5). Numerous studies have indicated that many genes, proteins and cell-autonomous signaling pathways have important roles in the chemoresistance of PC patients (6-9). Further analysis of these signaling pathways revealed that $\mathrm{Wnt} / \beta$-catenin signaling played important roles in drug exclusion from cells, changes in the enzymes metabolizing drugs, or the increased cell resistance to stress and apoptosis (4). The activation of Wnt/ $\beta$-catenin signaling was found to stimulate angiogenesis and regulate the cell cycle and apoptosis (10).

Among the multiple genetic and cytogenetic alterations that characterize human tumors, great emphasis has been recently given to epigenetic events, such as DNA methylation or histone acetylation (11). Alterations in histone acetyltrans- 
ferase (HAT) or histone deacetylase (HDAC) activity occur in numerous cancers and have prompted the search for pharmacological agents capable of inhibiting these enzymes. HDAC inhibitors show a very low toxicity in vivo and have become a new class of chemotherapeutic agents against cancer $(12,13)$. Trichostatin A (TSA) is one of the HDAC inhibitors (HDACi) and promotes histone hyperacetylation and strongly induces apoptosis by altering the expression of various apoptotic genes (14). However, in our previous study, gradually increasing concentrations of TSA in the PC cell line Panc-1 induced apoptosis. We found that MDR of the Panc-1 cells increased and the apoptotic effects of TSA decreased. Meanwhile, Wnt/ $\beta$-catenin signaling became highly activated. The precise mechanisms of HDACi-mediated chemosensitization, with regard to specific signal pathways/networks, remain somewhat speculative.

Based on these findings, we used a series of approaches to ascertain whether wnt-c59 inhibits Wnt/ $\beta$-catenin signaling. In conclusion, TSA-resistant Panc-1 cells (Panc-1/TSA) were re-sensitized to TSA.

\section{Materials and methods}

Reagents. TSA, dimethyl sulfoxide (DMSO), propidium iodide (PI) and Annexin V-FITC apoptosis detection kits were purchased from Sigma-Aldrich (St. Louis, MO,USA). Dulbecco's modified Eagle's medium (DMEM), fetal bovine serum (FBS), trypsin-EDTA, penicillin, streptomycin and TRIzol were obtained from Gibco-BRL (Invitrogen, Grand Island, NY, USA). TCF, E-cadherin, and MMP-9 antibodies were obtained from Cell Signaling Technology, Inc. (Beverly, MA, USA). Antibodies against Frizzled $7, \beta$-catenin and phospho- $\beta$-catenin were purchased from Abcam (Cambridge, UK). GAPDH, c-myc and horseradish peroxidase (HRP)-conjugated goat anti-rabbit secondary antibodies were obtained from Bio-World Co. (Nanjing, China). Reagents were used at the following working concentrations: $10 \mu \mathrm{mol} \cdot \mathrm{l}^{-1}$ of TSA; $10 \mu \mathrm{mol} \cdot 1^{-1}$ of Wnt/ $\beta$-catenin signaling inhibitor wnt-c59 (Gene Operation Inc., St. Joseph, MI, USA).

Cell lines and culture conditions. The cell line Panc-1/TSA was obtained from Dr Liu Biao (Wenzhou Medical University). Monolayer cultures of Panc-1/TSA cells were maintained in DMEM supplemented with $10 \% \mathrm{FBS}, 100 \mathrm{U} / \mathrm{ml}$ penicillin, and $100 \mu \mathrm{g} / \mathrm{ml}$ streptomycin at $37^{\circ} \mathrm{C}$ in a humidified chamber of 95\% air and $5 \% \mathrm{CO}_{2}$, and the medium was replaced every 2-3 days. When reaching subconfluency, the cells were harvested by trypzination, and recultured according to the experimental requirements. Cells were treated with TSA $\left(10 \mu \mathrm{mol} \cdot \mathrm{l}^{-1}\right)$ or wnt-c59 $\left(10 \mu \mathrm{mol} \cdot \mathrm{l}^{-1}\right)$ in $2 \mathrm{ml}$ of medium for $24 \mathrm{~h}$.

Real-time PCR analysis. Total cellular RNA was extracted from the different groups using TRIzol reagent (Invitrogen). Subsequently, mRNA was reverse transcribed to singlestranded cDNA using the ReverTra Ace ${ }^{\circledR}$ RT kit (Toyobo Co., Ltd., Osaka, Japan) according to the manufacturer's instructions. The cDNAs obtained were amplified by PCR using ABI 7500 Thermal Cycler Dice ${ }^{\mathrm{TM}}$ real-time system (Applied Biosystems, USA). The thermal cycler protocols included $3 \mathrm{~min}$ at $95^{\circ} \mathrm{C}$ and 40 cycles of $5 \mathrm{sec}$ at $95^{\circ} \mathrm{C}$ and $30 \mathrm{sec}$ at $60^{\circ} \mathrm{C}$.
The primer sequences are listed in Table I. For each sample, relative mRNA expression levels were derived from the ratio of their expression to GAPDH expression as an internal standard using the $2^{-\Delta \Delta \mathrm{Ct}}$ method (15).

Western blot analysis. Cells were harvested and lysed in lysis buffer containing protease inhibitors after the treatment with wnt-c59 and/or TSA, as required. The lysates were centrifuged at $12,000 \mathrm{xg}$ for $15 \mathrm{~min}$ at $4^{\circ} \mathrm{C}$. The supernatant was collected, and protein concentrations were determined by BCA protein assay according to the manufacturer's instructions. Primary antibodies were diluted 1:500-1:1,000 in 5\% skimmed milk with TBS-T [10 mM Tris- $\mathrm{HCl}$ (pH 8.0), $150 \mathrm{mM} \mathrm{NaCl}$ and $0.1 \%$ Tween-20]. Protein samples were solubilized and boiled in SDS sample buffer for $5 \mathrm{~min}$ and then separated using $12 \%$ SDS-PAGE at $200 \mathrm{~V}$ for $1 \mathrm{~h}$. Subsequently, the separated proteins were transferred to a polyvinylidene difluoride membrane. Following incubation in blocking solution consisting of 5\% skimmed milk in TBS-T for $1 \mathrm{~h}$ at room temperature and overnight incubation at $4^{\circ} \mathrm{C}$ with the primary antibodies including Frizzled $7, \beta$-catenin, phospho- $\beta$-cantenin, c-myc, TCF and GAPDH, the membrane was washed and then probed with respective horseradish peroxidase-conjugated secondary antibodies for $1.5 \mathrm{~h}$ at room temperature. After washing three times with TBS-T, hybridization was visualized using the ECL chemiluminescence detection system (Kodak). Expression levels of the proteins were compared to the control based on the relative intensities of the bands.

Immunofluorescent staining for assessment of cell morphology. Panc-1/TSA cells were incubated for $24 \mathrm{~h}$ using the desired method. After three washings with PBS, cells were fixed in $4 \%$ paraformaldehyde for $10 \mathrm{~min}$ at room temperature. The fixed cells were permeabilized with PBS containing $0.3 \%$ Triton X-100 for $10 \mathrm{~min}$. After soaking the cells in $10 \%$ goat serum (Solarbio, Beijing, China) with PBS for $30 \mathrm{~min}$, they were treated with the anti-Frizzled 7 antibody (1:200) in PBS overnight. The cells were then washed with PBS three times followed by incubation at $37^{\circ} \mathrm{C}$ for $30 \mathrm{~min}$. The secondary antibody solution mixed with PBS was incubated for $1 \mathrm{~h}$ in a dark room. After washing cells three times with PBS, they were covered with an anti-fade medium containing the nuclear stain 4',6-diamidino-2-phenylindole (MultiSciences Biotech, Hangzhou, China). Subsequently, they were examined using a DM4000B automated upright microscope system (Leica, Wetzlar, Germany).

Drug combination studies. Panc-1/TSA cells were trypsinized and seeded in 96-well cell culture plates $\left(1 \times 10^{4}\right.$ cells/well in $100 \mu \mathrm{l}$ culture medium) for overnight adhesion. They were then treated with wnt-c59 and/or TSA, as required, for an additional $24 \mathrm{~h}$. Next, cells treated in each well were maintained with $10 \mu \mathrm{l}$ of CCK-8 (Dojin, Japan) and $100 \mu \mathrm{l}$ DMEM at $37^{\circ} \mathrm{C}$ in $5 \% \mathrm{CO}_{2}$ for $2 \mathrm{~h}$. The optical density (OD) of each well was then measured using the Varioskan microplate reader (Thermo, USA) at a 450-nm wavelength. The cell inhibitory rate was calculated according to the following equation: The cell inhibitory rate $=1-[(\mathrm{OD}$ experiment - OD blank $) /(\mathrm{OD}$ control - OD blank)] x 100\%. We used the Zhengjun Jin method to determine whether the two drugs interact (16). The general 
Table I. The primers used in the real-time PCR.

\begin{tabular}{lll}
\hline Gene & \multicolumn{1}{c}{ Sense $\left(5^{\prime}-3^{\prime}\right)$} & \multicolumn{1}{c}{ Antisense (5'-3') } \\
\hline$\beta$-catenin & GCCAAGTGGGTGGTATAGAGG & GCGGGACAAAGGGCAAGA \\
Frizzled 7 & AGATCTGCGTGGGCCAGAAC & AGCGCCTCTCCTCCTCCTTA \\
GSK3 $\beta$ & AGACGCTCCCTGTGATTTATG & AACAAGAGGTTCTGCGGTTT \\
TCF & AGGCAGGGAGAATGCGACTC & GCGTTGAGGTTGGTGCCTTC \\
cyclin D1 & AGGCGGAGGAGAACAAACAG & TGAGGCGGTAGTAGGACAGGA \\
c-myc & CCACCACCAGCAGCGACT & CTTGACCCTCTTGGCAGCA \\
MDR1 & AGGTTCTGGGAAGATCGC & CCTCAAAGAGTTTCTGTATGGTA \\
GAPDH & TCCCATCACCATCTTCCAGG & GATGACCCTTTTGGCTCCC \\
\hline
\end{tabular}

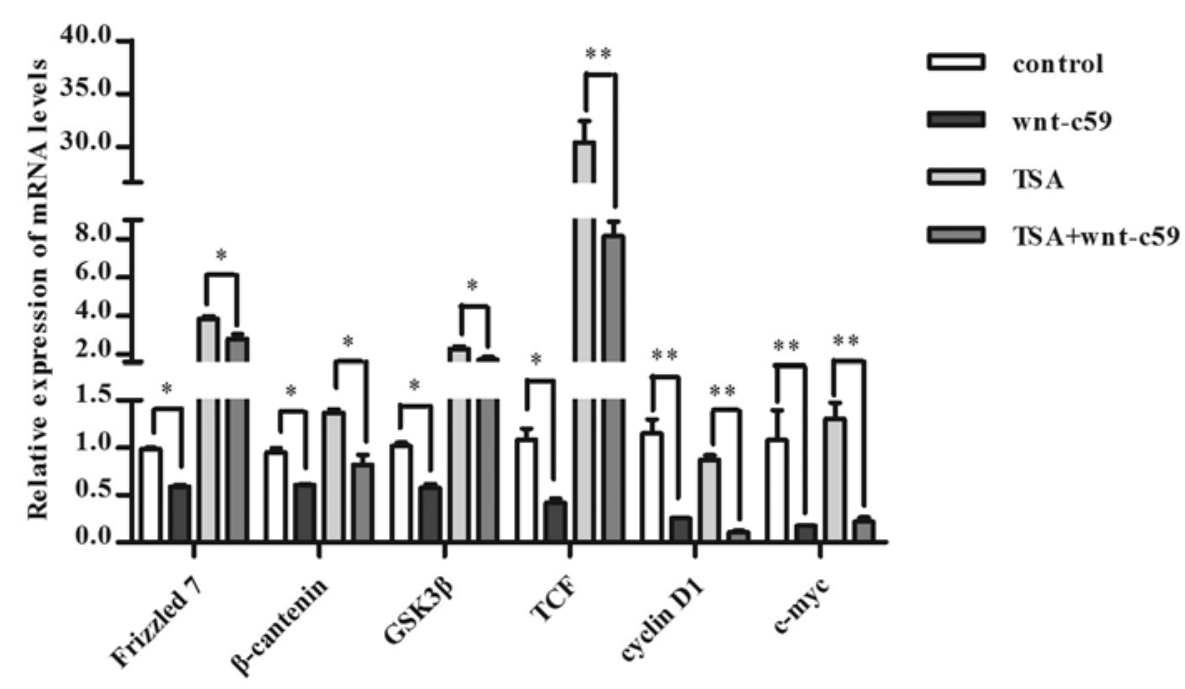

Figure 1. Expression of genes associated with the Wnt/ $\beta$-catenin signaling pathway detected by real-time RT-PCR. The mRNA expression levels of Frizzled 7 , $\beta$-catenin, GSK3 $\beta$, TCF, cyclin D1 and c-myc were significantly decreased after wnt-c59 and/or TSA treatment for $24 \mathrm{~h}$. The data are represented as the mean \pm SD of at least three independent experiments. ${ }^{*} \mathrm{P}<0.05 ;{ }^{* *} \mathrm{P}<0.01$.

equation is given by $q=E_{a+b} /\left(E_{a}+E_{b}-E_{a} \times E_{b}\right)$, where $E_{a}$ and $\mathrm{E}_{\mathrm{b}}$ in the denominator are the cell inhibition rates of TSA and wnt-c59 alone, respectively, whereas $\mathrm{E}_{\mathrm{a}+\mathrm{b}}$ in the numerator is the cell inhibitor rate of TSA and wnt-c59 in combination. $\mathrm{q}<0.85, \mathrm{q} \sim 0.85-1.15$, or $\mathrm{q}>1.15$ generally indicates an antagonistic, an additive, or a synergistic effect, respectively.

Flow cytometry. We used a monoclonal P-glycoprotein (P-gp) antibody (CD243, antibodies-online; Ebioscience, San Diego, CA, USA; 1:50) and the Annexin V apoptosis detection kit (Sigma-Aldrich). We added wnt-c59 and/or TSA into the Panc-1/TSA cells in the desired groups, collected $1-5 \times 10^{5}$ cells by centrifugation, and resuspended cells in $500 \mu 1$ of $1 \mathrm{X}$ binding buffer. A single-cell suspension was mixed with anti-CD243 at a ratio of $50: 1$ and stained for $30 \mathrm{~min}$ at $4^{\circ} \mathrm{C}$ or with $5 \mu \mathrm{l}$ of Annexin V-FITC and $10 \mu \mathrm{l}$ of propidium iodide for $5 \mathrm{~min}$ in the dark. Cells were washed with PBS. CD243-positive cells and apoptotic cells were detected by flow cytometry. All experiments were repeated three times.

Real-time cell analysis (RTCA) experiments. The migratory ability of the Panc-1/TSA cells was assessed by xCELLigence real-time cell analyzer (RTCA) DP device, which was placed in a humidified incubator at $37^{\circ} \mathrm{C}$ with $5 \% \mathrm{CO}_{2}$. Panc-1/TSA cells (Roche Applied Science, Basel, Switzerland) were seeded into the upper chamber of a two-chamber instrument separated by a porous membrane (CIM-plate 16). The cells either attached or migrated directly through the pores to the bottom side of the membrane. In either device, this increased the electrical impedance of integrated gold microelectrodes. The electrical impedance was displayed as a dimensionless parameter termed cell index. The cell index represents the capacity of cell migration, and the slope of the curve can be related to the migration velocity of the Panc-1/TSA cells. The cell index thus reflects the tumor cell migratory capacity. Prior to cell seeding the bottom side of the wells from the upper chamber of the CIM-plate 16 was coated with $30 \mathrm{ml}$ of collagen I. Furthermore, 40,000 cells/well suspended in culture medium containing $10 \%$ FBS were then seeded into the upper chamber according to the manufacturer's manual (17). The cell index was determined every $15 \mathrm{~min}$ for up to $24 \mathrm{~h}$ using RTCA software (ver. 1.2; Roche Diagnostics).

Statistical analysis. All data are expressed as mean values \pm SD. Statistical analysis was conducted using SPSS 13.0 software. The analysis of variance (ANOVA) was used to test 

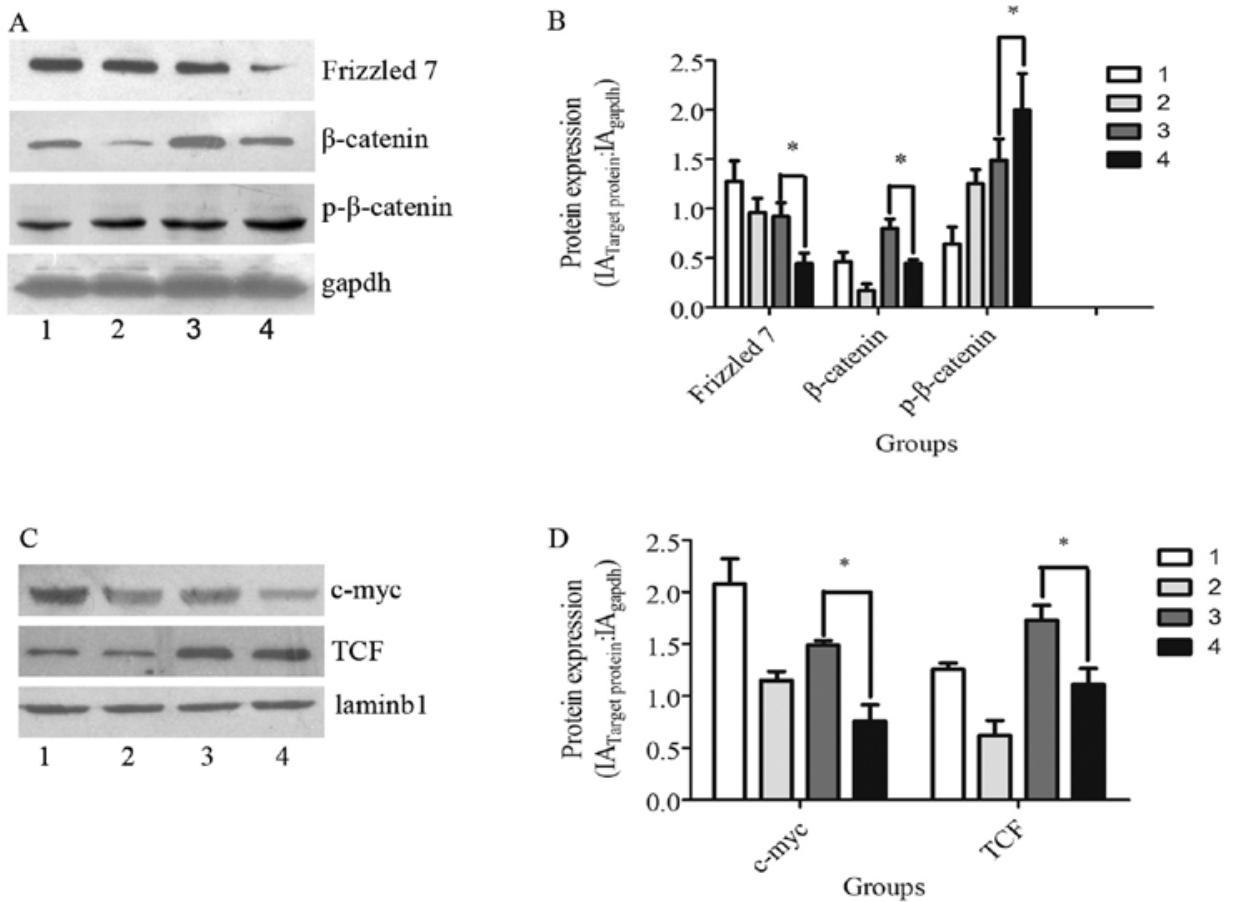

Figure 2. Protein expression of Frizzled 7, phosphorylated- and non- phosphorylated- $\beta$-catenin, TCF and c-myc measured by western blotting in Panc-1/TSA cells. (B and D) were the semiquantitative results of A and C, respectively. Lane 1: control group; lane 2: wnt-c59 group; lane 3: TSA group; lane 4: TSA+ wnt-c59 group. The data was represented by the mean \pm SD of at least three independent experiments ${ }^{*} \mathrm{P}<0.05$, TSA group compared to TSA+ wnt-c59 group.

the difference in the means between groups. Differences were considered statistically significant at $\mathrm{P}<0.05$.

\section{Results}

Downregulation of the expression levels of mediators of Wnt/ $\beta$ catenin signaling following wnt-c59 treatment. To determine the effects of TSA on mRNA expression of the mediators in Panc-1/TSA cells in the presence or absence of wnt-c59, we analyzed the level of mRNAs by real-time PCR. The results revealed that treatment with $10 \mu \mathrm{mol} \cdot \mathrm{l}^{-1}$ wnt-c59 significantly decreased mRNA expression levels of Frizzled $7, \beta$-catenin, downstream glycogen synthase kinase $3 \beta$ (GSK3 $\beta$ ) and T cell factor/lymphoid enhancer-binding factor 1 (TCF/LEF1). Consistently, transcriptional targets downstream of this pathway, such as c-myc and cyclin D1, were downregulated (Fig. 1). We confirmed these changes at the protein level by western blotting. As shown in Fig. 2, the protein levels of Frizzled 7, $\beta$-catenin, TCF and c-myc were decreased, correlating with the trend found for mRNA levels following wnt-c59 treatment. Immunofluorescence staining was performed to detect the cellular localization of the ligand-receptor Frizzled 7 protein, which revealed a membranous accumulation in the non-wnt-c59 treatment groups compared to the wnt-c59 treatment groups (Fig. 3). Our findings suggest that the inhibition of Wnt/ $\beta$-catenin activity in the Panc-1/TSA cells was due to downregulation of the expression of Wnt ligands, their receptors and modulators of Wnt signaling.

Interaction of TSA and wnt-c59 in the Panc-1/TSA cells. The results of the CCK-8 assay showed that the cell inhibitory rates of the wnt-c59 group, TSA group and TSA+wnt-c59 group were

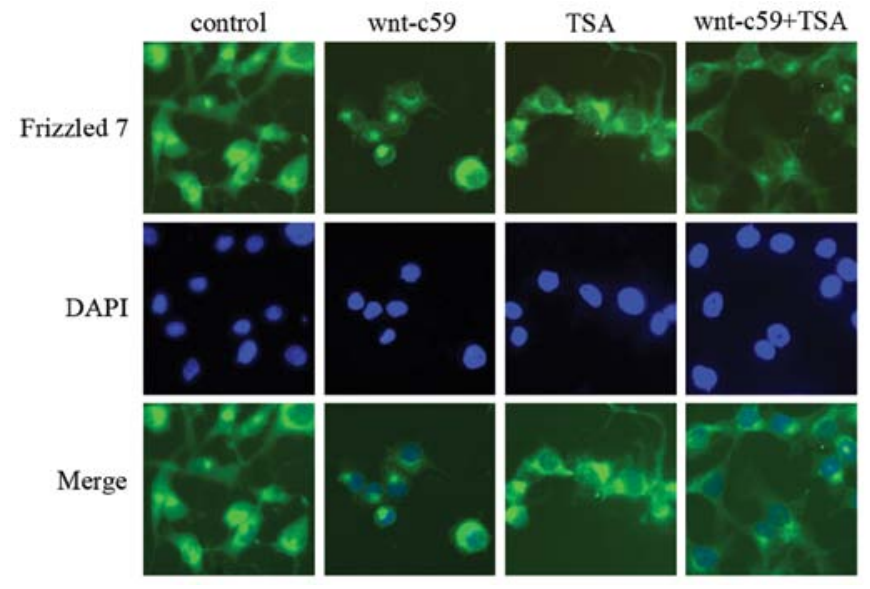

Figure 3. Expression of the ligand-receptor Frizzled 7 detected by immunofluorescence analysis. Minimal staining (membranous expression of Frizzled 7) was observed in Panc-1/TSA cells of wnt-c59 treated groups, whereas cells of non-wnt-c59 treated groups showed high levels of fluorescent staining readily distinguished from background. Zoom, x400.

$0.0028 \pm 0.0455,0.1960 \pm 0.0455$ and $0.2541 \pm 0.0059$, respectively. According to a method previously reported (16), $\mathrm{q}=1.282$ $>1.15$, indicated that the combination of TSA and wnt-c59 had a synergistic effect on inducing Panc-1/TSA cell apoptosis.

Effect of wnt-c59 on the apoptosis of Panc-1/TSA cells. To investigate the effect of wnt-c59 on the apoptosis of Panc-1/TSA cells, we detected cell apoptosis rates using flow cytometry. The cell apoptosis rates of the control group, wnt-c59 group, TSA group, and TSA+wnt-c59 group were $13.86 \pm 1.47,11.94 \pm 3.32,20.97 \pm 5.62$ and $37.24 \pm 8.2 \%$, respec- 

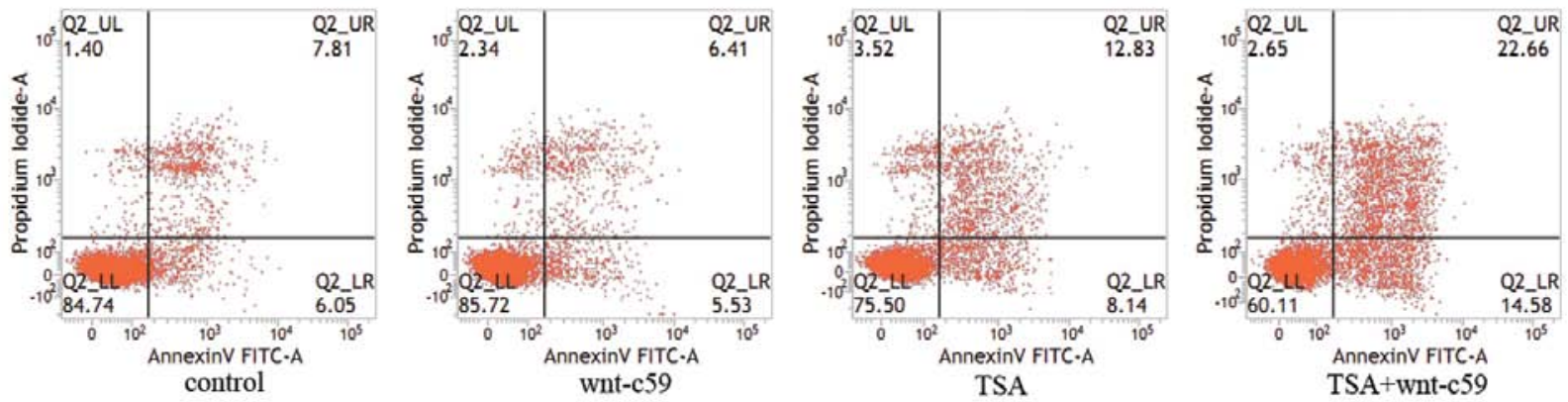

Figure 4. Induction of apoptosis by wnt-c59and/or TSA treatment. Panc-1/TSA cells were treated with wnt-c59 and/or TSA for 24 h. The percentage of apoptosis was calculated by Annexin $\mathrm{V}^{+}$(early apoptosis) and Annexin $\mathrm{V}^{+} /$propidium iodide ${ }^{+}$staining (late apoptosis).

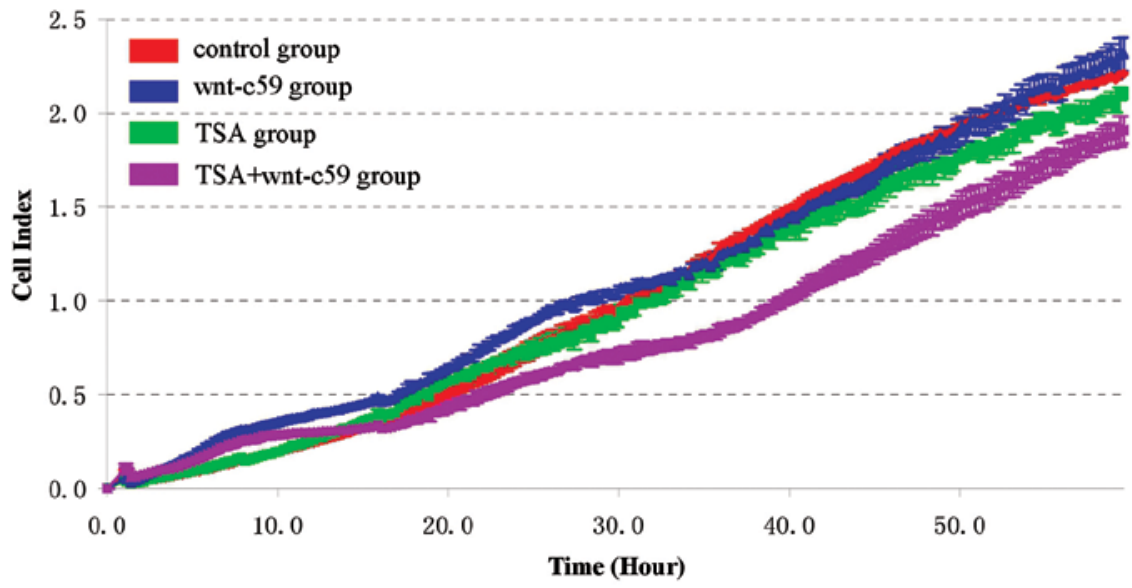

Figure 5. Real-time measurement of the cell migration in the Panc-1/TSA cells using the xCELLigence DP system. Panc-1/TSA cells were stimulated with wnt-c59 and/or TSA in serum-reduced medium during the assay as indicated by the color code. At 24, 36 and $48 \mathrm{~h}$ time points, differences between the TSA+wnt-c59 (purple curve) and the TSA treatment group (green curve) were significant $(\mathrm{P}<0.05)$ but not between the control and the wnt-c59 group.

Table II. Migration data of various groups measured by RTCA.

\begin{tabular}{|c|c|c|c|c|c|c|c|c|c|c|c|c|}
\hline \multirow[b]{2}{*}{ Time-interval (h) } & \multicolumn{3}{|c|}{ Control group } & \multicolumn{3}{|c|}{ Wnt-c59 group } & \multicolumn{3}{|c|}{ TSA group } & \multicolumn{3}{|c|}{ TSA+Wnt-c59 group } \\
\hline & Mean CI & SD & $\mathrm{CV}(\%)$ & Mean CI & SD & $\mathrm{CV}(\%)$ & Mean CI & SD & $\mathrm{CV}(\%)$ & Mean CI & SD & $\mathrm{CV}(\%)$ \\
\hline $24^{\mathrm{a}}$ & 0.7075 & 0.0393 & 5.5 & 0.7144 & 0.031 & 4.3 & 0.8579 & 0.0172 & 2 & $0.5694^{\mathrm{a}}$ & 0.0207 & 3.6 \\
\hline $36^{\mathrm{a}}$ & 1.2842 & 0.0257 & 2 & 1.2155 & 0.0282 & 2.3 & 1.2533 & 0.0194 & 1.5 & 0.8541 & 0.025 & 2.9 \\
\hline $48^{\mathrm{a}}$ & 1.8633 & 0.0105 & 0.5 & 1.6994 & 0.045 & 2.6 & 1.8315 & 0.0485 & 2.6 & 1.4153 & 0.0642 & 4.5 \\
\hline
\end{tabular}

Mean cell index (CI) represents the capacity for cell migration. $\mathrm{CV}$ represents the well-to-well variations. $\mathrm{SD}$, standard deviation. ${ }^{\text {a }}<<0.05$.

tively $(\mathrm{P}<0.05)$. The results of FACS detection showed that the apoptosis induced by the combination of TSA and wnt-c59 was significantly increased compared to the treatment with TSA or wnt-c59 alone (Fig. 4).

Reduced metastatic ability and reversal of EMT of Panc-1/TSA cells after treatment with the combined chemotherapeutics. We investigated the effect of inhibition of the $\mathrm{Wnt} / \beta$-catenin pathway on migratory ability. The results of RTCA showed that the cell indices of the control group, wnt-c59 group, TSA group, and TSA+wnt-c59 combination group were $1.2842 \pm 0.0257$, $1.2155 \pm 0.0282,1.2533 \pm 0.0194$ and $0.8541 \pm 0.0250$, respec- tively $(\mathrm{P}<0.05)$ (Fig. 5; Table II). In accordance, MMP-9 protein in the wnt-c59 treatment groups decreased compared to the non-wnt-c59 treatment groups. Meanwhile, the results of the western blotting indicated that the level of E-cadherin and vimentin protein, characteristic markers of EMT, was upregulated and downregulated, respectively (Fig. 6).

Inhibition of the Wnt/ $\beta$-catenin pathway decreases the expression of the MDR gene and protein. Various mechanisms contribute to MDR. One of the best-studied mechanisms is the increased expression of P-gp protein, which acts as a drug efflux pump lowering the intracellular concentration 
A

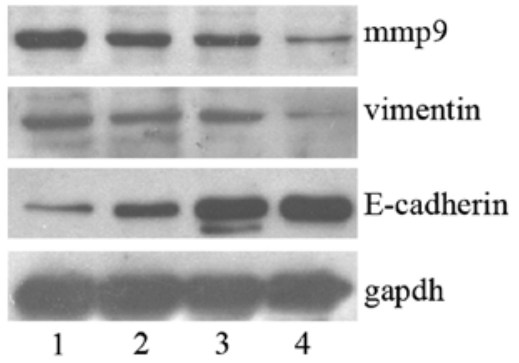

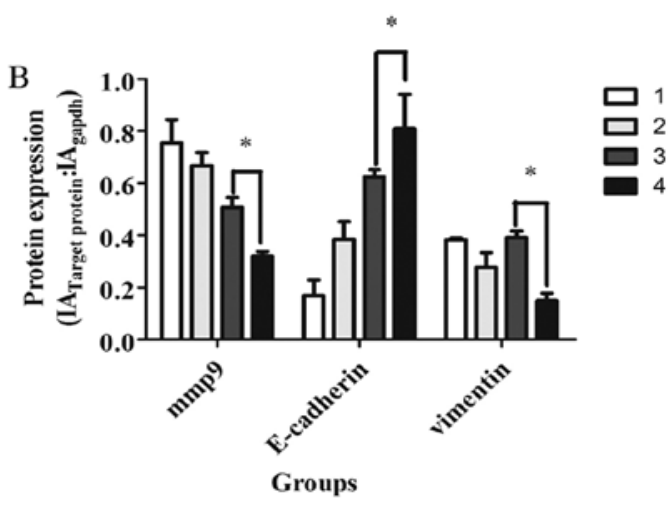

Figure 6. Effect of the inhibition of Wnt/ $\beta$-catenin on markers of EMT and cell migration. Panc-1/TSA cells in the wnt-c59 treatment groups exhibited lower expression levels of MMP-9 and vimentin proteins and higher expression levels of E-cadherin protein associated with EMT compared to the non-wnt-c59 treatment groups. A representative western blot is shown. (B) Semiquantitative results of A. Lane 1, control group; lane 2, wnt-c59 group; lane 3, TSA group; lane 4, TSA+wnt-c59 group. The data are represented as the mean \pm SD of at least three independent experiments. ${ }^{*} \mathrm{P}<0.05$, TSA group compared to the TSA+ wnt-c59 group.

A

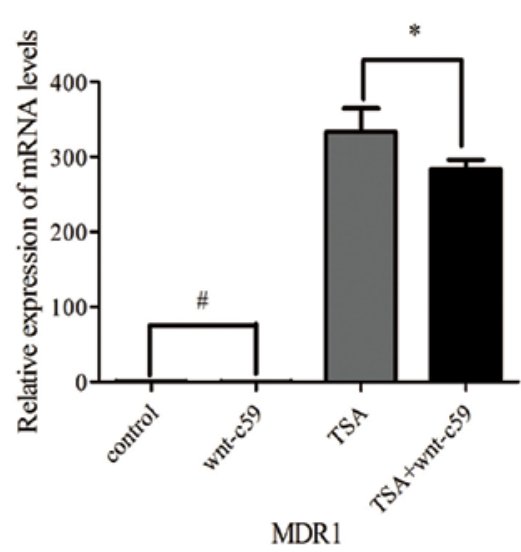

B
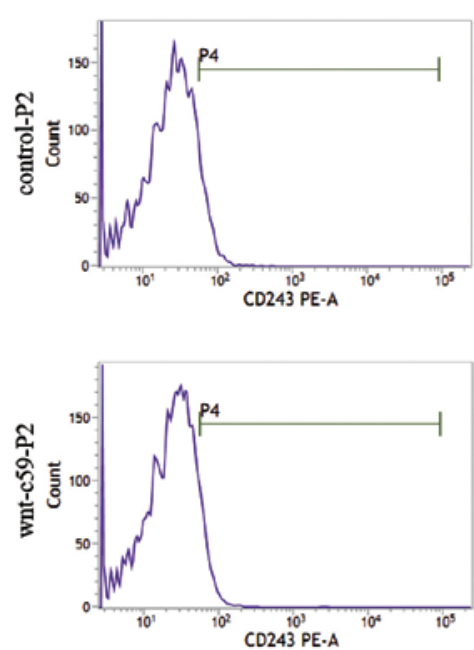
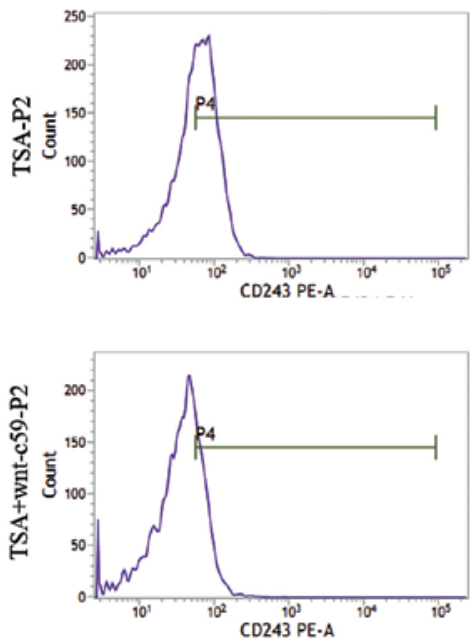

Figure 7. Downregulation of the MDR1 gene and P-gp protein in the Panc-1/TSA cells. (A) Real-time PCR demonstrated that the MDR1 transcript level was decreased after wnt-c59 treatment compared to that in the non-wnt-c59 treatment group. (B) FACS demonstrated that P-gp protein was downregulated after wnt-c59 treatment compared to that without wnt-c59 treatment.

Table III. Data of the P-gp protein results detected using FACS.

\begin{tabular}{|c|c|c|c|c|c|c|}
\hline Groups & Events & $\%$ Parent & $\%$ Grandparent & $\%$ Total & FSC-A mean & SSC-A mean \\
\hline Control & 720 & 8.53 & 7.20 & 7.20 & 158,412 & 139,773 \\
\hline Wnt-c59 & 704 & 7.96 & 7.04 & 7.04 & 131,582 & 128,787 \\
\hline TSA & 4,429 & 55.15 & 44.29 & 44.29 & 116,918 & 128,910 \\
\hline TSA+Wnt-c59 & 1,890 & 24.56 & 18.9 & $18.9^{\mathrm{a}}$ & 138,996 & 147,827 \\
\hline
\end{tabular}

${ }^{\mathrm{a}} \mathrm{P}<0.05$, TSA+wnt-c59 group compared with the TSA group.

of cytotoxic drugs (18). We detected expression of the MDR gene (MDR1) by real-time PCR. The results revealed that chemoresistant gene MDR1 in the wnt-c59 treatment groups had a lower expression compared to the levels in the nonwnt-c59 treatment groups $(\mathrm{P}<0.05$, Fig. 7A). We confirmed a similar change in the expression of chemoresistant protein P-gp using FACS (Fig. 7B; Table III).

\section{Discussion}

The aggressive and deadly biological characteristics of pancreatic cancer (PC) commonly result in the inability of the patients to undergo curative resection after diagnosis and thus chemotherapy becomes preferred treatment $(19,20)$. Unfortunately, PC often develops intrinsic resistance to continued chemo- 
therapy (21). Great advances in studying the mechanisms of tumor chemoresistance have been made. Studies have revealed that the $\mathrm{Wnt} / \beta$-catenin signaling pathway plays an important role in cell proliferation, differentiation, invasion and migration in many types of cancers. The Wnt/ $\beta$-catenin signaling pathway has become a frequently studied mechanism of cancer chemoresistance including that in PC $(22,23)$. However, very few studies have used chemical inhibitors of $\mathrm{Wnt} / \beta$-catenin signaling to overcome chemoresistance in PC therapy. We assumed that wnt-c59, one of the Wnt/ $\beta$-catenin signaling inhibitors, might inhibit $\mathrm{Wnt} / \beta$-catenin signaling, decreasing the expression of drug-resistant genes and proteins and the capacity of migration, reversing the EMT phenotype, and increasing the apoptosis induced by chemotherapeutic drugs in PC. Consequently, the drug susceptibility of Panc-1/TSA cells may be enhanced.

The Wnt/ $\beta$-catenin signaling pathway represents the cross-link between a series of signaling pathways. Classical Wnt/ $\beta$-catenin includes mainly extracellular factor wnt protein, transmembrane receptor protein $\beta$-catenin, and nuclear transcription factor TCF protein (24). $\beta$-catenin, called the CTNNB1 gene, is a type of adhesion molecule. $\beta$-catenin mediates intercellular adhesion and maintains cell morphology by binding to the intracellular domain of E-cadherin. $\beta$-catenin is also involved in Wnt signaling transduction. In the absence of Wnt signaling, $\beta$-catenin in the cytosol binds mostly to the intracellular domain of E-cadherin and is attached to the cytoskeleton protein actin by $\alpha$-cadherin to mediate intercellular adhesion. A part of $\beta$-catenin, which is complexed with adenomatous polyposis coli (APC) and Axin, is phosphorylated by casein kinase1 (CK1) as well as glycogen synthase kinase $3 \beta$ (GSK3 $\beta$ ) and is finally degraded. T cell factor/lymphoid enhancer-binding factor 1 (TCF/LEF1), due to the low $\beta$-catenin in the cytosol, is not activated since it binds to the transcription suppressor (25). In the presence of Wnt signaling, the signaling is initiated by Wnt proteins binding to the receptors of the Frizzled family on the cell surface. The activation of cytoplasmic degradation complex, comprising Axin, APC, GSK $3 \beta$ and CK1, is inhibited, leading to the accumulation of cytosolic $\beta$-catenin. Cytosolic $\beta$-catenin enters the nucleus and forms a complex with TCF/LEF1 to activate the transcription of Wnt target genes, such as MDR1, cyclin D1, c-myc, MMPs and IL-8 (10,26-29).

The downstream target genes of the Wnt/ $\beta$-catenin signaling pathway, such as MDR1, cyclin D1, c-myc, MMPs and IL- 8 , are related to chemoresistance. Overexpression of P-gp protein and the MDR1 gene, markers of MDR, is broadly known to limit the efficacy of chemotherapeutic drugs in inducing cancer cell apoptosis (30). Accumulating data indicate that several signaling pathways associated with the progression of tumor chemotherapy, such as Hedgehog, K-ras, TGF- $\beta$, Notch and Wnt/ $\beta$-catenin pathway signaling, play a pivotal role in the induction of MDR (31). Previous studies have demonstrated that the activation of $\mathrm{Wnt} / \beta$-catenin signaling contributes to the chemoresistance of human neuroblastomas or rat brain endothelial cells through upregulation of P-gp expression $(32,33)$. In a study of colon cancer the product of the MDR1 gene was upregulated with high expression of $\beta$-catenin (34). The data suggest that the role of $\mathrm{Wnt} / \beta$-catenin signaling is also involved in chemoresistance in PC (22). Our study results showed that the MDR1 gene and P-gp protein were downregulated after $\mathrm{Wnt} / \beta$-catenin signaling was inhibited by wnt-c59. With Wnt signaling activation, increasing nuclear $\beta$-catenin could promote high expression of c-myc and cyclin D1, which are involved in cell proliferation (35). Moreover, Kornmann et al and Qiao et al showed that chemoresistance genes and proteins were decreased after inhibition of expression of cyclin D1 $(36,37)$. In our study, after treatment with wnt-c59 in Panc-1/TSA cells, the transcriptional activity of Wnt/ $\beta$-catenin was inhibited and the apoptosis of Panc-1/ TSA cells was significantly increased as detected by FACS. Expression of cyclin D1 and c-myc was also downregulated. We speculate that one of the mechanisms reversing drug resistance involved inhibition of $\mathrm{Wnt} / \beta$-catenin signaling, which re-sensitized the cells to apoptosis and downregulated cyclin D1. At the same time, c-myc may decrease the expression of multiple chemoresistance genes and proteins.

In addition to multiple chemoresistance genes and proteins, accumulating evidence suggests that chemoresistance is associated with the acquisition of the EMT phenotype in PC cells $(38,39)$. Li et al found that PC cells that were sensitive to gemcitabine showed high expression of epithelial marker E-cadherin, whereas PC cells that were resistant to gemcitabine exhibited high expression of mesenchymal markers (including vimentin and $\mathrm{ZeB} 1$ ) at the gene and protein levels (40). These findings suggest that the EMT phenotype contributes to drug resistance in patients with PC. Moreover, drug-resistant cells from PC patients were more tumorigenic and had higher metastatic potential compared to cancer cells that were not drug resistant $(41,42)$. This propensity is largely responsible for the poor prognosis of PC with chemoresistance. Upon Wnt ligand binding to its receptors, $\beta$-catenin enters the nucleus and transcriptionally upregulates VEGF and IL-8. IL-8 can induce overexpression of MMP-2 and MMP-9, which can dissolve the extracellular matrix (ECM) in direct and indirect ways and serve as the initiator of proliferation and movement of endothelial cells $(33,43,44)$. In the present study, after inhibition of Wnt/ $\beta$-catenin signaling, the results of RTCA revealed that the migration ability of Panc-1/TSA cells in the TSA and wnt-c59 combination group was decreased significantly compared to the TSA alone group. We also found that the expression of E-cadherin protein was upregulated while the expression of vimentin and MMP-9 protein was downregulated. These results showed that the EMT phenotype was partly reversed after inhibition of $\mathrm{Wnt} / \beta$-catenin signaling.

In conclusion, inhibition of the Wnt/ $\beta$-catenin pathway can reverse drug resistance by downregulating the expression of multiple chemoresistance genes, proteins, and the EMT phenotype and by increasing cell apoptosis. Targeting Wnt/ $\beta$-catenin signaling could be useful for devising targeted therapeutic approaches for use in combination with conventional therapeutics to treat human PC. Compared to conventional approaches, these new treatments are expected to lead to better outcomes.

\section{Acknowledgements}

The authors are grateful for the funding from the Health Ministry of Zhejiang Province (Grant no. 11-ZC24 ), Zhejiang Provincial Top Key Discipline in Surgery, Administration of Traditional Chinese Medicine of Zhejiang Province (Grant 
no. 2012-XK-A28) and the Wenzhou Government (Grant no. Y20090028), China.

\section{References}

1. Akita H, Doki Y, Miyata H, et al: Clinical significance of the second cycle response to cisplatin-based chemotherapy as preoperative treatment for esophageal squamous cell carcinoma. J Surg Oncol 93: 401-409, 2006.

2. Badiglian Filho L, Oshima CT, De Oliveira Lima F, et al: Canonical and noncanonical Wnt pathway: a comparison among normal ovary, benign ovarian tumor and ovarian cancer. Oncol Rep 21: 313-320, 2009.

3. Batova A, Shao LE, Diccianni MB, et al: The histone deacetylase inhibitor AN-9 has selective toxicity to acute leukemia and drugresistant primary leukemia and cancer cell lines. Blood 100: 3319-3324, 2002.

4. Bordonaro M, Tewari S, Cicco CE, Atamna W and Lazarova DL: A switch from canonical to noncanonical Wnt signaling mediates drug resistance in colon cancer cells. PLoS One 6: e27308, 2011

5. Fournel M, Trachy-Bourget MC, Yan PT, et al: Sulfonamide anilides, a novel class of histone deacetylase inhibitors, are antiproliferative against human tumors. Cancer Res 62: 4325-4330, 2002.

6. Freese JL, Pino D and Pleasure SJ: Wnt signaling in development and disease. Neurobiol Dis 38: 148-153, 2010.

7. Ganesan A, Nolan L, Crabb SJ and Packham G: Epigenetic therapy: histone acetylation, DNA methylation and anti-cancer drug discovery. Curr Cancer Drug Targets 9: 963-981, 2009.

8. Gottesman MM, Fojo T and Bates SE: Multidrug resistance in cancer: role of ATP-dependent transporters. Nat Rev Cancer 2: $48-58,2002$.

9. Greenhalf $\mathrm{W}$ and Thomas A: Combination therapy for the treatment of pancreatic cancer. Anticancer Agents Med Chem 11: 418-426, 2011.

10. Griesmann H, Ripka S, Pralle M, et al: WNT5A-NFAT signaling mediates resistance to apoptosis in pancreatic cancer Neoplasia 15: 11-22, 2013.

11. Grimm M, Lazariotou M, Kircher S, et al: MMP-1 is a (pre-) invasive factor in Barrett-associated esophageal adenocarcinomas and is associated with positive lymph node status. J Transl Med 8: 99, 2010.

12. Hidalgo M and von Hoff DD: Translational therapeutic opportunities in ductal adenocarcinoma of the pancreas. Clin Cancer Res 18: 4249-4256, 2012.

13. Hong Y, Yang J, Wu W, et al: Knockdown of BCL2L12 leads to cisplatin resistance in MDA-MB-231 breast cancer cells. Biochim Biophys Acta 1782: 649-657, 2008.

14. Hung CC and Liou HH: YC-1, a novel potential anticancer agent, inhibits multidrug-resistant protein via cGMP-dependent pathway. Invest New Drugs 29: 1337-1346, 2011.

15. Inman GJ, Nicolas FJ, Callahan JF, et al: SB-431542 is a potent and specific inhibitor of transforming growth factor-beta superfamily type I activin receptor-like kinase (ALK) receptors ALK4, ALK5, and ALK7. Mol Pharmacol 62: 65-74, 2002.

16. Kornmann M, Danenberg KD, Arber N, Beger HG, Danenberg PV and Korc M: Inhibition of cyclin D1 expression in human pancreatic cancer cells is associated with increased chemosensitivity and decreased expression of multiple chemoresistance genes. Cancer Res 59: 3505-3511, 1999.

17. Le X, Shi Q, Wang B, et al: Molecular regulation of constitutive expression of interleukin-8 in human pancreatic adenocarcinoma. J Interferon Cytokine Res 20: 935-946, 2000.

18. Li Y, Van den Boom TG II, Kong D, et al: Up-regulation of miR-200 and let-7 by natural agents leads to the reversal of epithelial-to-mesenchymal transition in gemcitabine-resistant pancreatic cancer cells. Cancer Res 69: 6704-6712, 2009.

19. Liu X, Mazanek P, Dam V, et al: Deregulated Wnt/beta-catenin program in high-risk neuroblastomas without MYCN amplification. Oncogene 27: 1478-1488, 2008.

20. Liu Yong FY and Wang Hui: Synergistic augmentation of cisplatin-induced apoptosis in HeLa cells by rapamycin. Central China Med J 33: 300-303, 2009.

21. Livak KJ and Schmittgen TD: Analysis of relative gene expression data using real-time quantitative PCR and the 2(-Delta Delta C(T)) method. Methods 25: 402-408, 2001.

22. Long J, Zhang Y, Yu X, et al: Overcoming drug resistance in pancreatic cancer. Expert Opin Ther Targets 15: 817-828, 2011.

23. Loos M, Kleeff J, Friess H and Büchler MW: Surgical treatment of pancreatic cancer. Ann NY Acad Sci 1138: 169-180, 2008.
24. Masckauchan TN, Shawber CJ, Funahashi Y, Li CM and Kitajewski J: Wnt/beta-catenin signaling induces proliferation, survival and interleukin-8 in human endothelial cells. Angiogenesis 8: 43-51, 2005.

25. McCubrey JA, Steelman LS, Abrams SL, et al: Roles of the $\mathrm{RAF} / \mathrm{MEK} / \mathrm{ERK}$ and PI3K/PTEN/AKT pathways in malignant transformation and drug resistance. Adv Enzyme Regul 46: 249-279, 2006.

26. Mellor HR and Callaghan R: Resistance to chemotherapy in cancer: a complex and integrated cellular response. Pharmacology 81: 275-300, 2008.

27. Mimeault M, Hauke R and Batra SK: Recent advances on the molecular mechanisms involved in the drug resistance of cancer cells and novel targeting therapies. Clin Pharmacol Ther 83: 673-691, 2008.

28. Mimeault M, Johansson SL, Senapati S, Momi N, Chakraborty S and Batra SK: MUC4 down-regulation reverses chemoresistance of pancreatic cancer stem/progenitor cells and their progenies. Cancer Lett 295: 69-84, 2010.

29. Moore PS, Barbi S, Donadelli M, et al: Gene expression profiling after treatment with the histone deacetylase inhibitor trichostatin A reveals altered expression of both pro- and antiapoptotic genes in pancreatic adenocarcinoma cells. Biochim Biophys Acta 1693: 167-176, 2004.

30. Nakagami H, Soukupova H, Schikora A, Zarsky V and Hirt H: A Mitogen-activated protein kinase mediates reactive oxygen species homeostasis in Arabidopsis. J Biol Chem 281: 38697-38704, 2006.

31. Neoptolemos JP, Stocken DD, Bassi C, et al: Adjuvant chemotherapy with fluorouracil plus folinic acid vs gemcitabine following pancreatic cancer resection: a randomized controlled trial. JAMA 304: 1073-1081, 2010.

32. Osborn MT and Chambers TC: Role of the stress-activated/c-Jun NH2-terminal protein kinase pathway in the cellular response to adriamycin and other chemotherapeutic drugs. J Biol Chem 271: 30950-30955, 1996.

33. Qiao Q, Ramadani M, Gansauge S, Gansauge F, Leder G and Beger HG: Reduced membranous and ectopic cytoplasmic expression of beta-catenin correlate with cyclin D1 overexpression and poor prognosis in pancreatic cancer. Int J Cancer 95: 194-197, 2001.

34. Rocchi E, Khodjakov A, Volk EL, et al: The product of the ABC half-transporter gene ABCG2 (BCRP/MXR/ABCP) is expressed in the plasma membrane. Biochem Biophys Res Commun 271: 42-46, 2000.

35. Sayat R, Leber B, Grubac V, Wiltshire L and Persad S: $O$-GlcNAc-glycosylation of beta-catenin regulates its nuclear localization and transcriptional activity. Exp Cell Res 314: 2774-2787, 2008.

36. Shah AN, Summy JM,Zhang J,Park SI,Parikh NU and Gallick GE: Development and characterization of gemcitabine-resistant pancreatic tumor cells. Ann Surg Oncol 14: 3629-3637, 2007.

37. Siegel R, Ward E, Brawley O and Jemal A: Cancer statistics, 2011: the impact of eliminating socioeconomic and racial disparities on premature cancer deaths. CA Cancer J Clin 61: 212-236, 2011.

38. Su HY, Lai HC, Lin YW, et al: Epigenetic silencing of SFRP5 is related to malignant phenotype and chemoresistance of ovarian cancer through Wnt signaling pathway. Int J Cancer 127: 555-567, 2010.

39. Vaccaro V, Melisi D, Bria E, et al: Emerging pathways and future targets for the molecular therapy of pancreatic cancer. Expert Opin Ther Targets 15: 1183-1196, 2011.

40. van Hinsbergh VW, Engelse MA and Quax PH: Pericellular proteases in angiogenesis and vasculogenesis. Arterioscler Thromb Vasc Biol 26: 716-728, 2006.

41. Wang Z, Li Y, Kong D, et al: Acquisition of epithelial-mesenchymal transition phenotype of gemcitabine-resistant pancreatic cancer cells is linked with activation of the notch signaling pathway. Cancer Res 69: 2400-2407, 2009.

42. Yao J, Cai HH, Wei JS, et al: Side population in the pancreatic cancer cell lines SW1990 and CFPAC-1 is enriched with cancer stem-like cells. Oncol Rep 23: 1375-1382, 2010.

43. Yochum GS, Sherrick CM, Macpartlin M and Goodman RH: A beta-catenin/TCF-coordinated chromatin loop at MYC integrates 5' and 3' Wnt responsive enhancers. Proc Natl Acad Sci USA 107: 145-150, 2010.

44. Yuan RH, Jeng YM, Hu RH, et al: Role of p53 and $\beta$-catenin mutations in conjunction with CK19 expression on early tumor recurrence and prognosis of hepatocellular carcinoma. $\mathrm{J}$ Gastrointest Surg 15: 321-329, 2011. 